\section{Satisfaction of General Versus Specialized Continuity Clinic in Hematology Oncology Fellowship Training: A Survey}

\author{
Sama llyas ${ }^{1}$, Martina Murphy², Jennifer Duff ${ }^{2}$ and Julia Close ${ }^{2}$ \\ 1Department of Internal Medicine, University of Florida, Gainesville, FL, USA. ${ }^{2}$ Division of \\ Hematology and Oncology, University of Florida, Gainesville, FL, USA.
}

Journal of Medical Education and

Curricular Development

Volume 8: 1-5

(C) The Author(s) 2021

Article reuse guidelines:

sagepub.com/journals-permissions

DOI: $10.1177 / 23821205211025870$

(SSAGE

\begin{abstract}
BACKGROUND: At the University of Florida (UF), hematology-oncology (HO) fellows participate in 2 general types of continuity clinic as part of their fellowship training. One clinic, at the Veterans Hospital (VA), allows fellows to care for patients with a variety of hematology oncology diagnoses in a general clinic setting. The other clinic, located at the university site, is disease or system specific (such as breast or Gl clinic). Considerable research supports the value of continuity clinic in residency and fellowship training, but the differences in having a general versus specialized clinic for $\mathrm{HO}$ fellows have not been explored. The purpose of this study was to investigate the perceived differences of general versus specialized continuity clinics by recent $\mathrm{HO}$ graduates from UF. Specifically, we were interested in learning which features of a continuity clinic they felt were most impactful for their current clinical practice.
\end{abstract}

METHODS: An anonymous survey was sent to the last 6 graduating classes of HO fellows at UF, between years of 2013 and 2018 . The survey contained short demographic questions, followed by 5 open ended questions pertaining to the differing continuity clinic experiences. Graduates were asked about their opinions of both the general and specialized clinics during their training at UF. Survey responses were reviewed and coded for common themes by the authors.

RESULTS: Of 28 graduating fellows surveyed, 13 responded to the survey (response rate $46 \%$ ). In thematic review of survey responses, the most common themes that emerged concerned autonomy, level of supervision, and the diversity of the patient population. A majority of respondents felt they had more autonomy and personal responsibility at the VA general clinic, but less direct supervision than at the specialized clinics. They also believed they got a broader exposure to different disease types at the VA general clinic. Surveyed participants also commented on the quality of educational seminars and activities, preceptor expertise and teaching, and ability to observe cutting edge practice and clinical trials

CONCLUSIONS: Graduated oncology fellows from UF believe that there is a balance that exists between having autonomy and ownership of their patients versus having adequate supervision. Many believe that having "controlled autonomy" and "as much independence as is safe for patients" is key to a meaningful continuity clinic experience during oncology fellowship training

KEYWORDS: Continuity clinic, medical education, hematology oncology fellowship training

RECEIVED: March 5, 2021. ACCEPTED: May 28, 2021

TYPE: Original Research

FUNDING: The author(s) received no financial support for the research, authorship, and/or publication of this article.
DECLARATION OF CONFLICTING INTERESTS: The author(s) declared no potentia conflicts of interest with respect to the research, authorship, and/or publication of this article.

CORRESPONDING AUTHOR: Sama llyas, Department of Internal Medicine, University of Florida, Gainesville, FL, USA. Email: sama.ilyas@medicine.ufl.edu
Continuity clinic experiences have been established as a core constituent of many residency and fellowship curriculums. A continuity clinic is defined as a longitudinal outpatient experience, where a resident or fellow follows a patient panel over the course of their training. Research has shown the need for greater education in ambulatory and outpatient settings in general internal medicine, as graduating residents are often uncomfortable managing chronic problems. ${ }^{1,2}$ Thus, greater emphasis on outpatient and continuity care has been placed in many internal medicine residency programs.

While there is considerable literature regarding general internal medicine curriculum, there is less research that has explored hematology oncology (HO) fellowship training. The ACGME requires a "structured continuity ambulatory clinic experience that exposes [fellows] to the breadth and depth of medical oncology." 3 Current literature in oncology fellowship training has investigated integrating geriatric and palliative training into the curriculum, ${ }^{4,5}$ and there has also been research on the structure of oncology fellowship didactic seminars. ${ }^{6}$ However, continuity clinic experiences in oncology fellowship training have not yet been explored.

At the University of Florida (UF), oncology fellows participate in 2 general types of continuity clinic as part of their training. One clinic at the Veterans Hospital (VA) allows them to care for patients in a general clinic setting that encompasses a variety of hematology oncology diagnoses. The other clinic, located at the university site, is disease or system specific (such as breast or GI clinic). Differences in having a general versus specialized clinic for oncology fellows have not been explored. The purpose of this study is to investigate the perceived differences of general versus specialized continuity clinics by recent oncology graduates from UF, and what features of a continuity clinic they feel were most important for an effective and meaningful experience. 
Survey: Satisfaction of general versus specialized continuity clinic in oncology fellowship training

Population: graduated hematology oncology fellows from UF - last 6 years

Survey questions:

Which year did you graduate from fellowship?

[2013, 2014, 2015]

[2016, 2017, 2018]

Which describes your current practice environment?

[Academic]

[Community]

[Industry]

[Other - please describe]

What describes the majority of your practice?

[General hematology oncology clinic]

[Focused clinic; eg. breast, GI, leukemia]

[Equal time devoted to general and focused clinic]

1. What were the benefits of having continuity clinic at the VA (general clinic)?

2. What were the disadvantages of having continuity clinic at the VA (general clinic)?

3. What were the benefits of having a specialized continuity clinic at UF?

4. What were the disadvantages of having a specialized continuity clinic at UF?

5. In your opinion, what top three factors constitute a valuable continuity clinic experience for fellowship training?

Figure 1. Clinic Satisfaction Survey

\section{Method}

\section{Sample}

The sample size consisted of the most recent 6 graduating classes of hematology oncology fellows at the University of Florida, encompassing classes of 2013 to 2018. Two participants were excluded due to their involvement in this project, so a total of 28 graduates were surveyed. Of those 28 graduates, 13 completed the survey ( $46 \%$ response rate).

\section{Measures and procedures}

Survey questions were developed by the team in the form of short demographics and 5 open-ended questions (see Figure 1 for survey). The survey was designed using the Qualtrics software. Surveys were then sent to graduates $(\mathrm{N}=28)$ via email. Responses were collected anonymously.

\section{Analysis}

Analysis of the qualitative data derived from the surveys was completed using a coding system. After survey responses were collected, each member of the team individually coded the responses for common themes. Inductive coding style was used, where codes are derived from analyzing data and recognizing patterns, as opposed to a deductive style, where codes are pre-set. ${ }^{7,8}$ The team then met to compare for inter-coder reliability. Thus, responses were coded according to the hematology oncology fellow graduates' opinions on the 2 different types of continuity clinics that they experienced during their training. Using this method of coding allowed for an openended construction of themes that may be valuable to getting insight into underlying feelings and attitudes amongst oncology graduates. Access to these deeper insights, derived from free flow responses, may provide valuable feedback in regards to the continuity clinic training experience.

\section{Results}

A chart showing demographics of the survey respondents is shown in Figures 2 and 3. Sixty-nine percent of survey participants currently practice in a general hematology oncology setting, while $31 \%$ work in a focused clinic. Sixty-two percent of respondents work in a community setting, while $23 \%$ and $15 \%$ practice in academic and government settings, respectively.

The results from the surveys did not overwhelmingly support either a general or specialized continuity clinic. However, there were several themes brought up to contrast the 2 clinic settings. We will explore the major themes that arose from analyzing the responses.

\section{Autonomy}

Many participants brought up the idea of autonomy as being a critical determinant of their continuity clinic experience. 


\section{Practice Type}

\begin{tabular}{|l|r|}
\hline $\begin{array}{l}\text { General hematology } \\
\text { oncology }\end{array}$ & 9 \\
\hline $\begin{array}{l}\text { Focused clinic (e.g. } \\
\text { breast, Gl, leukemia) }\end{array}$ & 4 \\
\hline
\end{tabular}

General hematology oncology

Focused clinic (e.g. breast, GI, leukemia)

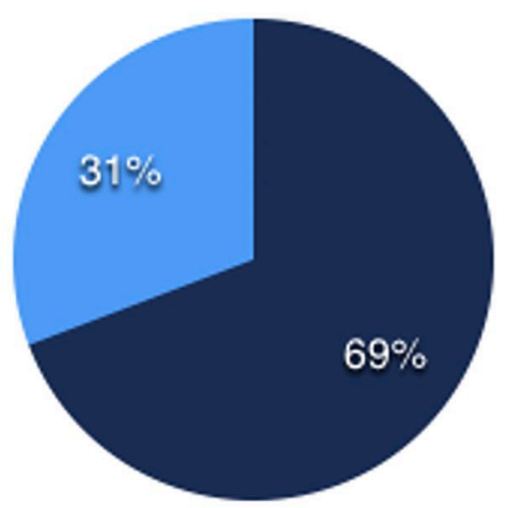

Figure 2. Practice Type

The idea of "controlled autonomy" was brought up by several participants, which encompasses having the ability to independently make decisions in a supervised setting. Participants believed that "having as much independence as is safe for patients" and "autonomy within reason to come up with your own treatment plans" was crucial to their experience. Autonomy was one of the major themes brought up, as respondents felt that having autonomy allowed them to take ownership of their patients, which in turn led them to have a more immersive experience.

\section{Immersion/responsibility}

Related to autonomy is the theme of responsibility: respondents who felt they had autonomy indicated they felt more directly responsible for their patient panel. Respondents felt that having "responsibility pushes [you] to be up to date and helps tremendously in practice" and that having "total immersion in patient care" facilitated this.

\section{Supervision}

Some respondents felt that there was too much supervision at times, at the cost of autonomy, while others believed "closer supervision [was] not always available" and that there was a "lack of direct supervision." Some respondents felt that they needed a different level of supervision as they progressed through their training, and that this was not necessarily reflected by their preceptors.

\section{Practice environment}

\begin{tabular}{|l|r|}
\hline Academic & 3 \\
\hline Community & 8 \\
\hline Industry & 0 \\
\hline Government & 2 \\
\hline
\end{tabular}

\section{Academic Industry Community
Government}

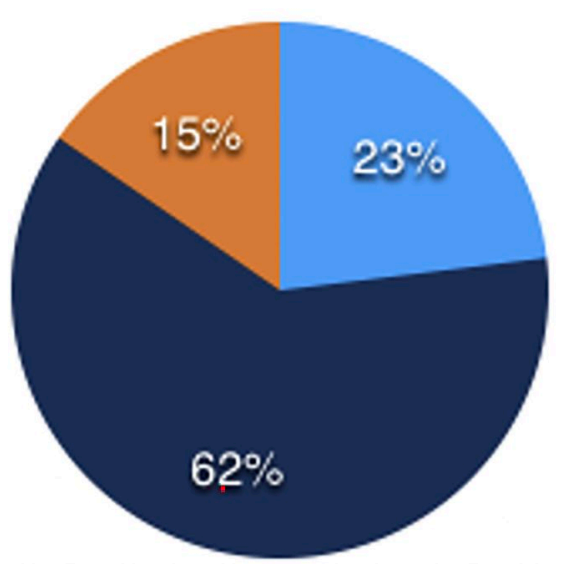

Figure 3. Practice Environment

\section{Diversity}

Many participants commented on diversity, or variety, as a factor in their clinic experiences. Diversity, in this situation, encompasses diversity of patient demographics (as most patients at the VA are male), but also diversity in terms of cases. Respondents felt that VA general clinics had "no exposure to female cancer patients" but specialty clinics did not allow for "getting as broad exposure" on a variety of diagnoses.

\section{Preceptor teaching}

One respondent reported that it "really helps to learn the specifics of treating one type of cancer from an expert who has years of knowledge" and that it "allows you to learn practice patterns."

\section{Volume}

Respondents felt that there should be a "right volume of patients" and that having an increased case-load facilitates fellows "to become accustomed to a very high volume practice."

\section{Transition to practice}

One respondent felt that having a continuity clinic at the VA general clinic "was the absolute most beneficial part of [their] 
fellowship training." They asserted that "learning how to manage [their] own panel, being responsible for orders, treatment plans, and interacting with the staff provided a critical foundation to build upon." They went on to comment that "transitioning into private practice, [they] felt very comfortable managing [their] own practice.”

\section{Educational seminars}

Participants commented on the differing quality of didactics they received at each clinic as being a factor. One respondent felt that increased quality of didactics was helpful to a good continuity clinic experience. Another respondent felt there should be "more push towards attending tumor boards presented by fellows."

\section{Cutting edge trials}

Participants commented on having exposure to the newest trials and keeping up to date as a valuable part of their training. Respondents felt that at the VA general clinic there was an "occasional limitation in drug availability and access to clinical trials," while the specialized clinic had more "cutting edge" clinical work, where they were able to "discuss clinical trials and the recent practice changing studies" with their preceptor.

\section{Discussion}

The current study provides a qualitative analysis of graduated fellows' opinions regarding their continuity clinic experiences at our institution. Using inductive coding allowed for an open ended construction of themes, from which we can make some valuable assumptions. We can infer from the survey results that there are a variety of factors that lead to a positive continuity clinic experience. Many of these factors are interrelated, such as the themes of autonomy, supervision, and responsibility.

Since many of these factors are related, there seems to exist a trade-off when 1 factor is favored over the other. A good example of this is the idea of autonomy versus supervision. Many fellows believed that having autonomy allowed them to become more immersed in the experience, and therefore feel a more personal responsibility toward their patients. However, this is contrasted by having adequate supervision by preceptors in order to ensure that the highest standards of patient safety are still met. Thus, there exists a balance between autonomy and supervision. This seems best solved by the idea of "controlled autonomy"-having enough autonomy as is safe for patients, with the least amount of direct supervision needed to ensure this.

An interesting sub-text to explore is whether the autonomy in question is true autonomy, or if it is perceived autonomy by the fellows. This idea could potentially be explored, since a likely hypothesis would be that autonomy in any form would encourage fellows to have more immersion and ultimately feel more personal responsibility. A continuity clinic may be the perfect set up to create an optimal controlled setting, where a fellow is able to immerse themselves into the practice, but with underlying guidance. This would describe a setting of "controlled autonomy," which would help with transition to practice as well.

One respondent brought up an interesting comment regarding supervision. They asserted that while they appreciated greater supervision earlier on in their training, supervision in their third year became overbearing. This brings up the idea that continuity clinic should conform to the fellow's level of experience in order to form an optimal longitudinal experience.

\section{Limitations}

There are several relevant limitations to this study. The greatest limitation may be biased responses. Many continuity clinic experiences may be influenced based on extraneous factors, such as the individual preceptors with whom the fellow works. Every preceptor is unique and can form a different learning environment. There are also some preceptor-fellow pairs that may work better than others. Having different preceptors in the different continuity clinics is a variable that was not controlled in this study.

Another limitation is the small sample size. While the response rate was just under 50\%, graduates that were more likely to complete this study may have had stronger feelings toward their experiences, either positive or negative, that motivated them to complete the survey.

\section{Implications}

The current study helps with the construction of an optimal hematology oncology fellowship continuity clinic experience. Based on the responses, it seems that it is not the type of clinic itself (general or specialized) that may be determinant, but the factors that make up those clinics. Neither the VA general clinic or the specialized clinics at the university site were perceived as superior to one another, but both highlight different strengths and weaknesses. Thus, we can optimize any continuity clinic experience by implementing factors that are known to maximize the benefits derived by the fellow. These identified factors can be used to strengthen a program's curriculum by being implemented in the continuity clinic. While our study was conducted in the setting of $\mathrm{HO}$ graduates, there may be further implications for other sub-specialty programs, as the themes that emerged were not specific to the care of patients with hematologic/ oncologic problems.

\section{Conclusions}

Graduated oncology fellows from UF believe that there is a balance that exists between having autonomy and ownership of their patients versus having adequate supervision. Many believe that having "controlled autonomy" and "as much independence as is safe for patients" is key to a 
meaningful continuity clinic experience during oncology fellowship training. Controlled autonomy helps facilitate total immersion and increased personal responsibility. Other important factors of a continuity clinic include variety/diversity of patient population and cases, patient volume, preceptor teaching and expertise, exposure to new trials, and quality of educational seminars. The many determinants that graduated fellows believe constitute a positive continuity clinic experience can be implemented into a program's curriculum to optimize every fellow's experience, which in turn will translate to a more positive transition to care.

\section{Acknowledgements}

Poster presented as ASCO in May 2019.

\section{Author Contributions}

All authors contributed equally to the manuscript.

\section{REFERENCES}

1. Sisson SD, Boonyasai R, Baker-Genaw K, Silverstein J. Continuity clinic satisfaction and valuation in residency training. J Gen Intern Med. 2007;22(12): 1704-1710.

2. Holmboe ES, Bowen JL, Green M, et al. Reforming internal medicine residency training. A report from the Society of General Internal Medicine's task force for residency reform. J Gen Intern Med. 2005;20:1165-1172.

3. Advanced Specialty Program Requirements for Graduate Medical Education in Medical Oncology. ACGME International. https://www.acgme-i.org/Portals/0/ Specialties/InternalMedicine/147InternalMedicineOncology07012016. pdf?ver=2017-09-18-092639-463

4. Eid A, Hughes C, Karuturi M, Reyes C, Yorio J, Holmes H. An interprofessionally developed geriatric oncology curriculum for hematology-oncology fellows. $J$ Geriatr Oncol. 2015;6:165-173.

5. Thomas RA, Curley B, Wen S, Zhang J, Abraham J, Moss AH. Palliative care training during fellowship: a national survey of U.S. hematology and oncology fellows. J Palliat Med. 2015;18:747-751.

6. Eid A, Hsieh P, Shah P, Wolff R. Cross-sectional longitudinal study of the academic half-day format in a hematology-oncology fellowship training program. BMC Med Educ. 2015;15:139.

7. Hesse-Biber S. Analyzing qualitative data with or without software. Boston University. https://www.bumc.bu.edu/crro/files/2010/07/Hesse-Bieber-4-10. pdf

8. Saldana J. An introduction to codes and coding. The Coding Manual for Qualitative Researchers. https://www.sagepub.com/sites/default/files/upm-binaries /24614_01_Saldana_Ch_01.pdf 